\title{
Role of Statistical Parameter in Digital Image Enhancement
}

\author{
Dhirendra Pal Singh \\ Computer Centre, University of Lucknow, Lucknow (U.P.) - 226007 \\ Email: dhirendrapal@yahoo.com
}

\begin{abstract}
There are several domains like medical, astronomical, hybrid techniques, remote sensing, pattern recognition, high resolution display etc., in which people want to get an image and store it so that the images may be used to extract some valuable information using image quality features in the future. Image quality estimation aims to evaluate the image information automatically by a computational model hence plays a significant role in digital image processing systems. In this paper to meet the need of accuracy and effectiveness for gray scale images, role of various image enhancement quality features are discussed.
\end{abstract}

Keywords - Mean Square Error(MSE), Signal to Noise Ratio(SNR), Structural Content(SC), Image Enhancement, Maximum Difference. Recall.

Date of Submission: Sep 25, 2019

Date of Acceptance: Mar 07, 2020

\section{INTRODUCTION}

Image processing technique[1][2] deals with efficient techniques to retrieve the information from images. If it is done digitally, it produces more advance and enhanced results. This technique involves various tasks like how to represent the image, how to examine the image, how to reproduce the image, and how to enhance the image. Overall it is the art of examining, identifying and judging the impact of the images so that one can be able to explore the significance of the image for study by the human interpreters. There are several domains like medical, astronomical etc., in which people want to get an image and store it so that the images may be used to extract some valuable information in the future. The field of digital image processing has experienced dramatic growth and increasingly widespread applicability in recent years. Digital Image Processing techniques can be applied on image with the help of digital computer to improve image quality for human perception and prepare the image for measurement of the features. The primary purpose of image processing is to convert image in to valuable information and this can be achieved by its statistical methods like PSNR, MSE, ISNR etc. Image quality estimation aims to evaluate the image information automatically by a computational model hence plays a significant role in digital image processing systems. In this paper to meet the need of accuracy and effectiveness for gray scale images, various image enhancement[3] quality features are explained.

\section{PERFORMANCE STATISTICAL}

\section{PARAMETER}

How to evaluate the enhancement result is an open problem. Image enhancement is intended to convert images into a form that makes the use of capabilities of human visual system to perceive information to their highest degree. The image quality after enhancement is evaluated mainly by a person's vision. This problem becomes more apparent when the enhancement algorithms are parametric in nature and one need to choose the best parameters, to choose the best algorithms among class of algorithms, or to automate the image enhancement procedures. The problem becomes especially difficult when an image enhancement procedure is used as a preprocessing step for other image processing purposes such as the diagnosis of diseases, object detection, classification, and recognition on sets of images. The greatest difficulty in image enhancement is to quantify the evaluation criteria for enhancement. A lot of research has been done in the field of Image Enhancement feature extraction in terms of measurement of visual quality given as below-

1. Structural Content

2. Improvement in Signal to Noise Ratio

3. Mean Square Error

4. Root Mean Square Error

5. Peak to Signal Noise Ratio

6. Maximum Difference

7. Precision

8. Recall

9. Accuracy

10. F-Score

\section{II.I STRUCTURAL CONTENT}

Structural Content (SC) may be defined as:

$$
S C=\sum_{m=1}^{M} \sum_{n=1}^{N} x(m, n)^{2} \div \sum_{m=1}^{M} \sum_{n=1}^{N} x^{*}(m, n)^{2}
$$

where :

$\mathbf{x}(\mathbf{m}, \mathbf{n})$ is Original Image,

$x^{*}$ is Segmented Image and

$\mathrm{M}, \mathrm{N}$ are number of pixels in rows and columns of image. 
Larger value of SC describes poor quality of the image and smaller value of the SC describes better quality of the image.

\section{II.II IMPROVEMENT IN SIGNAL TO NOISE RATIO}

For the purpose of objectively testing the performance of image we used three statistical measures improvement in signal to noise ratio(ISNR). The Improvement in signal to noise ratio(ISNR) may be defined as the improvement in the quality of the estimated image over the blurred image [4]

$$
I S N R=\|g-f\|^{2} /\left\|f-f^{\prime}\right\|^{2}
$$

where :

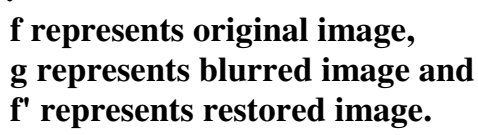

f represents original image, g represents blurred image and f' represents restored image.

Higher values of ISNR signify good results and lower values of ISNR represent poor results.

\section{II.III MEAN SQUARE ERROR}

Mean Square Error(MSE)[4] is another quality measure which quantitatively recognize the strength of error signal. MSE is frequently used because it has a simple mathematical structure and easy to implement. For a discrete image signal $x(m, n)$ and its approximation or segmented image $x^{*}(m, n)$, MSE may be defined mathematically :

$$
M S E=(1 \div M N)\left(\sum_{m=1}^{M} \sum_{n=1}^{N}\left(x(m, n)-x^{*}(m, n)\right)^{2}\right)
$$

where :

$x(m, n)$ is original image,

$x^{*}$ is Segmented Image and

$\mathrm{M}, \mathrm{N}$ are number of pixels in rows and columns of image.

Higher value of Mean Square Error (MSE) refers to the poor quality of image and the lower value of the MSE describe better quality of the image.

\section{II.IV ROOT MEAN SQUARE ERROR}

Root Mean Square Error (RMSE)[5] It is commonly used to compare the difference between the reference and fused images by directly computing the variation in pixel values. The combined image is close to the reference image when RMSE value is zero. RMSE is a good indicator of the spectral quality of fused image and may be defined as:

$$
R M S E=\sqrt{M S E}
$$

\section{II.V PEAK TO SIGNAL NOISE RATIO}

When different dynamic range images are being compared then MSE may be used in the form of PSNR[4][6]. PSNR is expressed in decibels $(\mathrm{dB})$. Peak to Signal Noise Ratio (PSNR) defined as :

$$
P S N R=10 * \log (255 * 255 / M S E)
$$

If Peak Signal to Noise Ratio (PSNR) have small value, it means the Image Quality is not good.

\section{II.VI MAXIMUM DIFFERENCE (MD)}

Another Image Quality Measures, Maximum Difference (MD), is the maximum of error signal. $\mathrm{MD}$ is the difference between the original image signal and segmented image signal. MD is mathematically defined as:

$$
M D=\operatorname{Max}\left(\left|x(m, n)-x^{*}(m . n)\right|\right)
$$

where :

$$
\begin{aligned}
& x(m, n) \text { is Original Image, } \\
& x^{*} \text { is Segmented Image and } \\
& M, N \text { are number of pixels in rows and columns } \\
& \text { of image. }
\end{aligned}
$$

Higher value of MD indicates lower quality image and lower value indicate good quality image.

\section{II.VII PRECISION, RECALL, ACCURACY AND F-SCORE}

Since Precision and Recall [7][8] individually measures the quality of information retrieval, therefore, we make use of a single measure, 'F-Score', to do the similar assessment.

\section{II.VII .I PRECISION}

Precision is defined as the ratio of correctly detected characters to sum of correctly detected characters plus false positives; where false positives / false alarms are those regions in the image which are actually not characters of a text, but have been detected by the algorithm as text.

\section{II.VII .II RECALL}

Recall is defined as the ratio of the correctly detected characters to sum of correctly detected characters plus false negatives; where false negatives / misses are those regions in the image which are actually text characters, but have not been detected by the algorithm.

\section{II.VII .III ACCURACY}

Accuracy measures the degree of exactness or fidelity and may be defined as the ratio of the sum of true positive 
$(T P)$ and true negative $(T N)$ to the sum of true positive, false positive (FP), true negative and false negative $(\mathrm{FN})$, which is expressed as :

Accuracy $=(\mathrm{TP}+\mathrm{TN}) /(\mathrm{TP}+\mathrm{TN}+\mathrm{FP}+\mathrm{FN})$.

\section{II.VII .IV F-SCORE}

F-score is the harmonic mean of Recall and Precision rates and expressed as :

F-Score $=(2 *$ Precision $*$ Recall $) /($ Precision + Recall $)$.

\section{Significance Of Statistical Paramater}

Statistical parameter plays significant role in feature extraction in digital image enhancement. A number of researches are available to demonstrate the importance of various statistical parameters. Some of the major contributions by various authors are described in this section as below.

In 2006, Ung-Keun Cho et.al.[9] proposed a method to improve the quality of fingerprint image by using the Genetic Algorithm; and performance of proposed method shows better results with the help of parameter 'Error rate' as shown in fig. 1 .

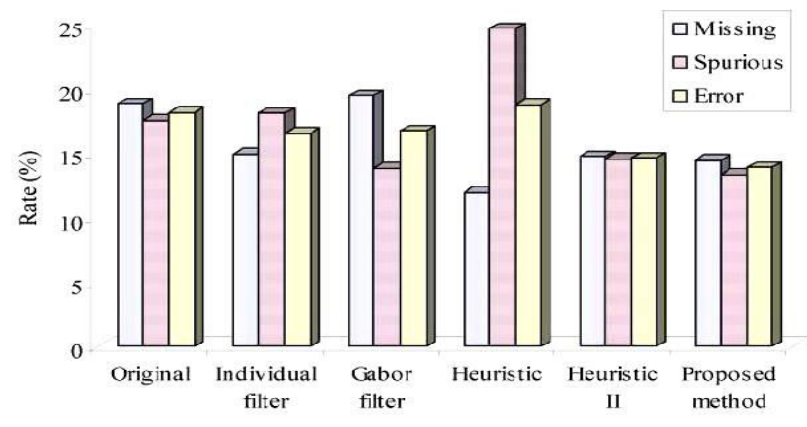

Fig. 1

S.A. Qureshi et.al [10] explain that image reconstruction has been done using Binary Genetic Algorithm(BGA) by evaluating various fitness function and the image quality has been measured by a variety of image quality matrices RMS

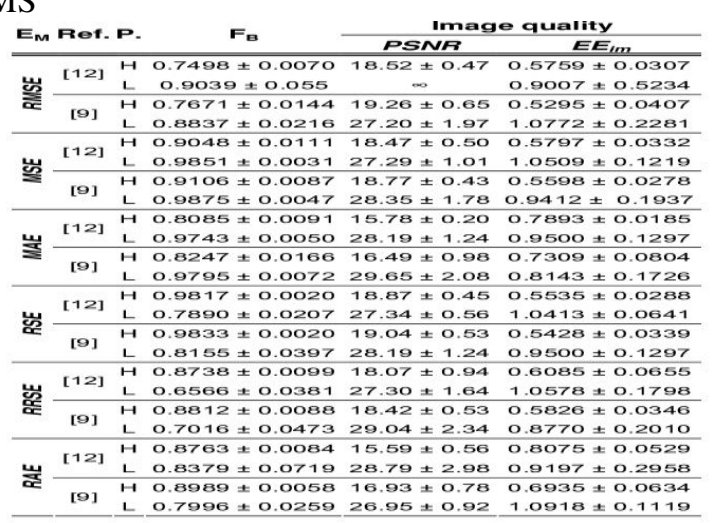

RMSE, MSE, MAE etc. as shown above.

In 2011 R. Chandrasekaran[11] proposed a method for text extraction in images on the basis of Morphological based operation and performance of proposed method to get best feature extraction can be evaluated using Statistical parameter Precision, Recall and F-Score

In year 2003, Jong Bae Kim[12] proposed an efficient technique for image restoration on the basis of Genetic algorithm. For the performance of proposed method, Improvement in Signal to Noise Ratio (ISNR) is used as shown in fig. 3 .

\begin{tabular}{lccc}
$(\mathrm{dB})$ & Nonconstraint & Laplacian constraint & Proposed method \\
\hline Synthetic 1 & 16.41 & 17.01 & 17.78 \\
Synthetic 2 & 9.41 & 9.98 & 10.96 \\
\hline
\end{tabular}
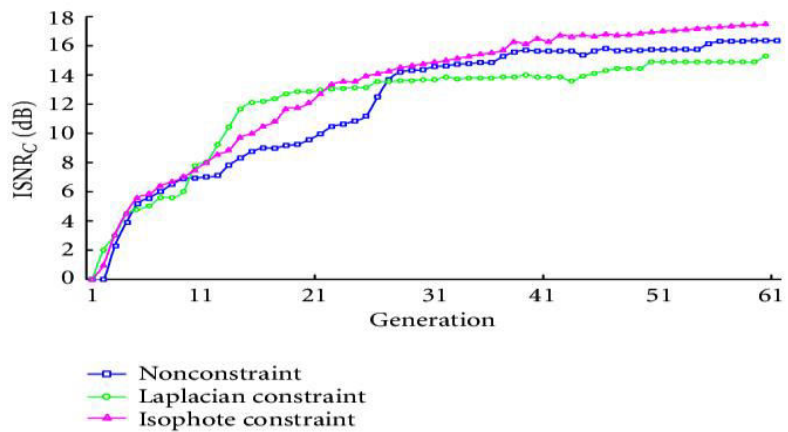

(a)

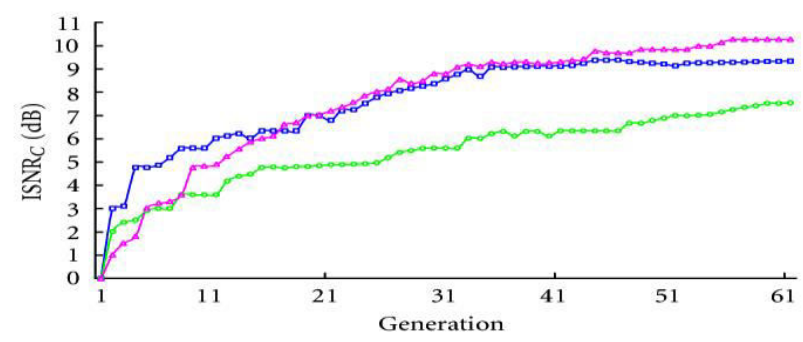

$\rightarrow$ Nonconstraint
$\therefore$ Laplacian constraint
$\therefore$ Isophote constraint

(b)

Fig. 3

In their experimental results he explains that value of ISNR in terms of restoration results improves as the number of generation increases (as shown in fig. 3).

In the year 2004 Sonja Gregic [13] paper entitled 'Reliability of objective picture quality' explains the significance of various statistical measures MSE, PSNR MD etc. in still image compression system, has been investigated. In this paper three different image compression system JPEG, JPEG2000 and SPIHT are considered for objective and subjective picture quality assessment as shown in fig. 4. 
Fig. 4
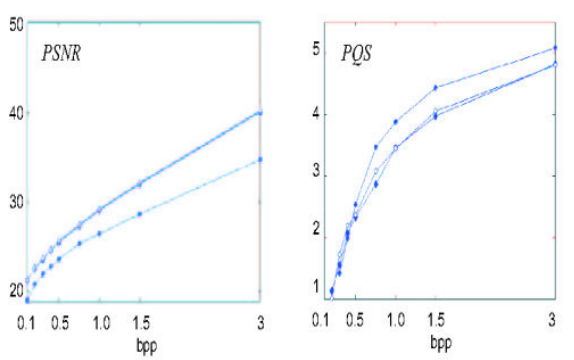

(a) Baboon
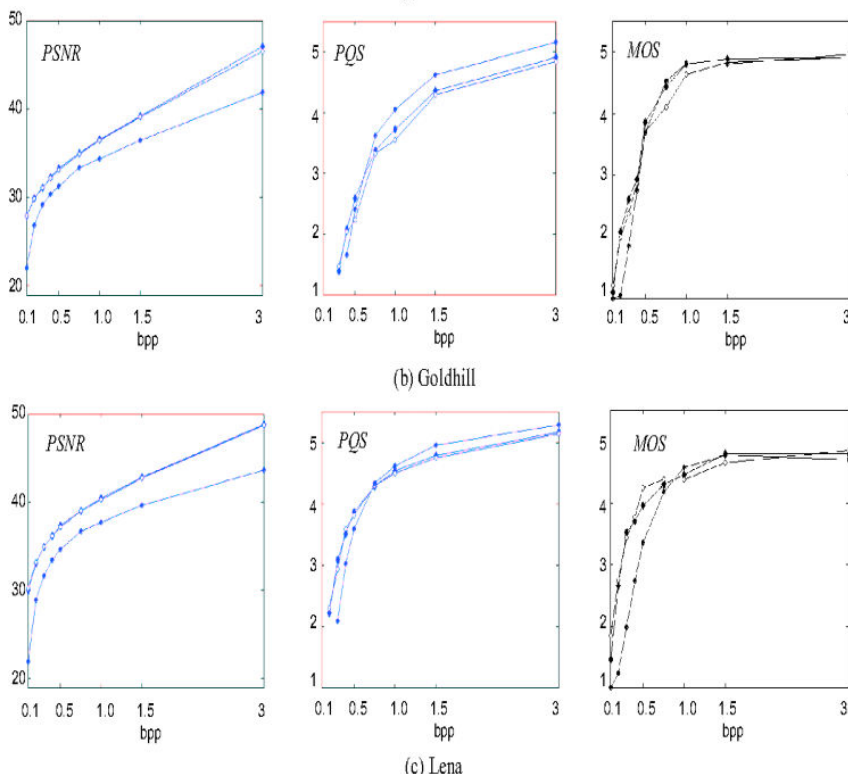

\section{Conclusion}

The primary purpose of digital image processing is to convert image in to valuable information and this can be achieved by its statistical parameters. This paper explores the role of different types of image quality metrics for getting the best quality of an image. The above research content is by no means in-depth. Further library search and other sources are needed to make the material collected more comprehensive.

\section{ACKNOWLEDGEMENT}

The Author is grateful to all the above discussed researchers that help in bringing out the useful information.

\section{REFERENCES}

[1] R. C. Gonzalez, R. E. Woods, Digital Image Processing, Addison-Wesley, 1987.

[2] A. K. Jain, Fundamentals of Digital Image Processing, Prentice-Hall Inc., 1989.

[3] R. Korfhage, Information Storage and Retrieval, Willey, NewYork, 1997.

[4] I. A.,. B. S.,. K. S. Ismail Avcibas, 'Statistical Evaluation of Quality Measures in Image Quality Compression', Journal of Electronic Imaging, 2002.
[5] Zoran, L.F., 2009. 'Quality Evaluation of Multiresolution Remote Sensing Image Fusion' U.P.B. Sci. Bull., Ser. C 71, 38-52.

[6] A. L. M. Jean-Bernard Martens, 'Image dissimilarity', Signal Processing, vol. 70, no. 3, pp. 155-176, 1998.

[7] R. Korfhage, Information Storage and Retrieval, Willey, NewYork, 1997.

[8] Avcbas I, Sankur B, Sayood K., 'Statistical evaluation of image quality measures' Journal of Electronic Imaging. 2002; 11(2):206-23

[9] U. K. Cho, J. H. hong and S. B. Cho, 'Evolutionary Singularity Filter Bank Optimization for Figerprint Image Enhancement' Journal of Electrical Engineering. LNCS 3907,2006; 55(1-2):380-390.

[10] S. A. Qureshi, S. M. Mirza and M. Arif, 'Fitness Function Evaluation for Image Reconstruction Using Binary Genetic Algorithm for Parallel Ray Transmission Tomography' International Journal of Computer Science and Network Security, Jan. 2007, 7(1):132-137.

[11] R Chandrasekaran and RM. Chandrasekaran, 'Morphology Based Text Extraction in Images' International Journal of Computer Science and Technology. Oct.-Dec.2011; 2(4):103-107.

[12] J. B. Kim and H. J. Kim, 'GA-Based Image Restoration by Isophote Constraint Optimization' EURASIP Journal of Applied Signal Processing, 2003, 3, 238-243.

[13] Sonja Grgic, Mislav Grgic and Marta Mrak, 'Reliability of Objective Picture Measures' Journal of Electrical Engineering. 2004; 55(1-2):3-10.

\section{Author Biography}

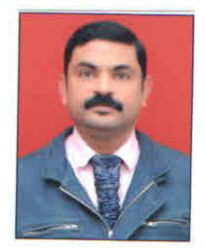

Mr. Dhirendra Pal Singh received his undergraduate Degree in Engineering in Computer Science \& Information Technology (B.Tech (CSIT)) from I.E.T., Bareilly in 1999. He received his PostGraduate Degree in Computer Science, M.Sc.(CS), MCA and Ph.D. in Computer Science in the area of Image Processing Applications. Presently he is working in Computer Centre, University of Lucknow, Lucknow (U.P.). 